\title{
Smart Logistics - Technologiekonzepte und Potentiale
}

\author{
Helmut Zsifkovits und Manuel Woschank \\ Lehrstuhl für Industrielogistik, Montanuniversität Leoben, Leoben, Österreich \\ Eingegangen 6. November 2018; angenommen 27. November 2018; online publiziert 14. Januar 2019
}

\begin{abstract}
Zusammenfassung: Die intelligente Steuerung von Supply Chains ist eine wesentliche Voraussetzung für die Erschließung der Potenziale neuer digitaler Technologien. Im folgenden Beitrag wird Smart Logistics als Komponente von Industrie 4.0 und in ihren Zusammenhängen zu anderen Komponenten dargestellt. Es werden Technologiekonzepte der Smart Logistics beschrieben. Darauf aufbauend werden Nutzungspotenziale und mögliche weitere Entwicklungen analysiert. Als Ergebnis eines internationalen Projektes werden weiters die Voraussetzungen dargestellt, die in Unternehmen für die Einführung von Smart Logistics gegeben sein müssen.
\end{abstract}

Schlüsselwörter: Smart Logistics, Smart Supply Chain Management, Industrie 4.0, SME 4.0

\section{Smart Logistics-Technology Concepts and Potentials}

Abstract: The intelligent control of supply chains is an essential prerequisite for exploiting the potential of new digital technologies. In the following paper, Smart Logistics will be discussed as a component as well as in its relationship to other components within an Industry 4.0 framework. Technology concepts of Smart Logistics are described. Based on this, potential applications and possible further developments are analyzed. As a result of an international project, the requirements that must be met in companies for the introduction of smart logistics are presented.

Keywords: Smart logistics, Smart supply chain management, Industry 4.0, SME 4.0


Lehrstuhl für Industrielogistik,

Montanuniversität Leoben, Erzherzog-Johann-Straße 3, 8700 Leoben, Österreich helmut.zsifkovits@unileoben.ac.at

\section{Einleitung}

Digitalisierung steht für den zunehmenden Einsatz vernetzter digitaler Systeme in allen Bereichen der Gesellschaft. Der Begriff der Industrie 4.0 umfasst heterogene Ansätze der Digitalisierung in der industriellen Wertschöpfung. Die Einsatzbereiche reichen hierbei von der Produktentwicklung, Konstruktion und Fertigung bis hin zur Steuerung der Materialflüsse mit dem Ziel, die Wettbewerbsfähigkeit durch erhöhte Transparenz, Agilität, Adaptivität und Flexibilität wesentlich zu erhöhen [1].

Die zentralen Konzepte von Industrie 4.0 sind Vernetzung und Selbststeuerung [2].

An den Knoten des Produktionsnetzwerkes, den produzierenden Einheiten, den Lagerorten und Umschlagspunkten werden über Sensoren und andere Systeme der Erfassung Informationen gesammelt. Produkte, Bauteile und Stoffe tragen die Information über ihre Natur, Beschaffenheit und ihren Status an sich. Diese Knoten und Objekte sind vernetzt und kommunizieren miteinander.

Über Regeln und Algorithmen kann eine teilweise oder vollständige Selbststeuerung geschaffen werden. Produktionsschritte und -reihenfolgen können jederzeit dynamisch angepasst werden. Lagerbestände werden ständig überprüft und können bei Bedarf automatisch nachbeschafft werden.

Dafür erforderlich sind neben einer laufenden Identifikation von Objekten und Zuständen, einer informationstechnischen Vernetzung von Anlagen und Produkten auch autonome Systeme des Materialflusses sowie die Systeme der Steuerung.

Die intelligente Steuerung von Supply Chains ist eine wesentliche Voraussetzung für die Erschließung der Potenziale neuer digitaler Technologien [3]. Der folgende Beitrag widmet sich der Digitalisierung von Supply Chains, der Nutzung von digitalen Technologien in inner- und überbetrieblichen Materialflüssen.

Smart Logistics wird als Komponente von Industrie 4.0 und in ihren Zusammenhängen zu anderen Komponenten dargestellt. Es werden Technologiekonzepte der Smart Logistics beschrieben. Darauf aufbauend werden Nutzungspotenziale und mögliche weitere Entwicklungen analysiert. 
Anhand eines Modells von Supply Chains werden die beschriebenen Ansätze eingeordnet. Als Ergebnis eines internationalen Projektes werden weiters die Voraussetzungen dargestellt, die in Unternehmen für die Einführung von Smart Logistics gegeben sein müssen.

\section{Smart Logistics als Element eines Rahmenkonzeptes Industrie $\mathbf{4 . 0}$}

Kagermann definiert Industrie 4.0 als "die technische Integration von CPS in die Produktion und die Logistik sowie die Anwendung des Internets der Dinge und Dienste in industriellen Prozessen - einschließlich der sich daraus ergebenden Konsequenzen für die Wertschöpfung, die Geschäftsmodelle sowie die nachgelagerten Dienstleistungen und die Arbeitsorganisation [4]." Hier werden explizit die Produktion und Logistik als Kernbereiche angesprochen, Geschäftsmodelle und andere Unternehmensfunktionen sind nach dieser Definition indirekt betroffen. Somit ist "Smart Logistics" ein Kernelement von Industrie 4.0.

Hermann et al. [5] identifizierten aufgrund einer quantitativen Textanalyse und einer qualitativen LiteraturanaIyse von akademischen und praktischen Veröffentlichungen unter Anwendung der nominalen Gruppentechnik vier Gestaltungsprinzipien für Industrie 4.0. Diese sind: Vernetzung, dezentrale Entscheidungen, transparente Informationen und technische Assistenz.

Das Industrie-4.0-Framework von Capgemini Consulting beschreibt acht Werttreiber, zugeordnet den vier Säulen Smart Solutions, Smart Innovation, Smart Supply Chains und Smart Factory. In Erweiterung der Perspektive von Kagermann werden hier die Produkte und Dienstleistungen sowie die Prozesse der Innovation als eigenständige Elemente dargestellt. Diese vier Säulen sind als Faktoren zur Wachstumssteigerung und Effizienzsteigerung eingebettet in ein organisatorisches Framework, bestehend aus einem agilen Betriebsmodell, Personalführung, Change-Management, Governance und Prozesse sowie der digitalen Infrastruktur. Aus der Zusammenführung und Erweiterung dieser Ansätze ergibt sich das in Abb. 1 dargestellte Smart Logistics Framework [6, 7].
Smart Logistics umfasst somit intelligente und schlanke Lieferketten, aufbauend auf agilen Kooperationsnetzwerken und einer organisatorischen Vernetzung. Eine informatorische Vernetzung erfolgt durch Systeme der IKT, Datennetze, Sensorik und Technologien der automatischen Identifikation. Autonome Transportmittel in Verbindung mit automatisierten Lägern und Umschlaginfrastrukturen ermöglichen eine weitgehende oder vollständige Selbststeuerung.

\section{Technologiekonzepte und Potenziale der Digitalisierung im Rahmen der Smart Logistics}

In der Folge wird auf die Technologiekonzepte eingegangen, die der Industrie 4.0 zugrunde liegen. Diesbezüglich gibt es in der Literatur sehr divergente Klassifizierungsansätze. Capgemini Consulting nennt als technologische Treiber für Industrie 4.0 Cloud-Computing, Mobile Technologien, Robotik, Advanced Analytics, Machine-to-MachineKommunikation, Soziale Medien und 3D-Druck [6]. Diese Klassifikation ist jedoch eher unspezifisch und umfasst in einer sehr weiten Perspektive Technologien, die eine Basis für Systeme der Smart Produktion und Smart Logistics bilden, aber nicht unbedingt zu deren Charakterisierung dienen.

In der Folge beschrieben werden die Konzepte der cyberphysischen Systeme (CPS), das Internet der Dinge (IoT) sowie das Physical Internet; diese sind spezifische Merkmale von Smart Logistics.

"Cyber-physische Systeme (CPS)“ sind physische Objekte oder Strukturen, wie Produkte, Geräte, Gebäude, Verkehrsmittel, Produktionsanlagen, Logistikkomponenten und mehr, die eingebettete Systeme enthalten und dadurch kommunikationsfähig werden [8]. Diese Systeme sind über lokale und globale digitale Netze miteinander verbunden [9] und umfassen Sensoren wie auch Aktoren, welche in geschlossenen Regelkreisen interagieren [10].

Cyber-physische Systeme können ihre Umwelt unmittelbar mit ihrer Sensorik erfassen, sie mit Hilfe weltweit verfügbarer Daten und Dienste auswerten und speichern, und sie können mit Hilfe von Aktoren auf die physikalische
Abb. 1: Smart Logistics Framework

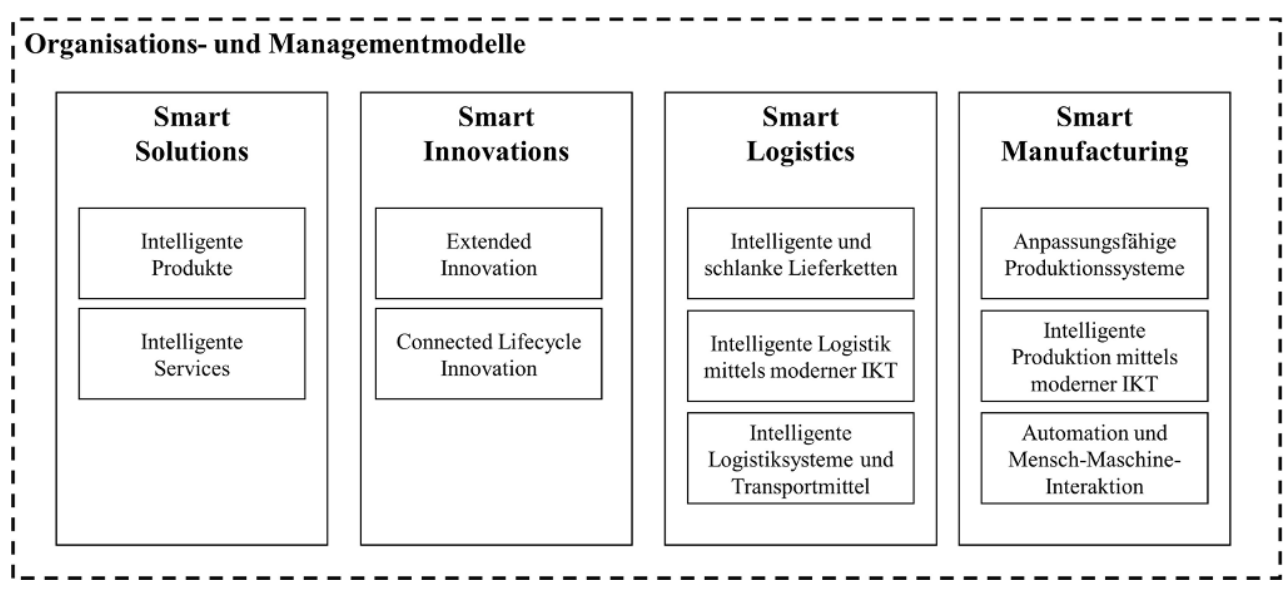


Welt einwirken. Sie können sich vernetzen und autonom und dezentral im Sinne selbstähnlicher Produktionsfraktale sich eigenständig optimieren. Die intelligente Fabrik (Smart Factory) interagiert mit Menschen oder Maschinen und ist in der Lage, sich dezentral selbst echtzeitnah zu organisieren [8]. Ein virtuelles Abbild der Realität wird permanent mit Hilfe der Echtzeitdaten aktualisiert; über diesen „digitalen Zwilling" wird die reale Welt mit der virtuellen Welt synchronisiert. Dies ist die Basis, um das Internet der Menschen mit dem Internet der Dinge und dem Internet der Dienste zu verbinden.

Der Begriff "Internet der Dinge (IoT)“ als integraler Bestandteil von CPS wird stark mit der Technologie der RadioFrequency Identification (RFID) assoziiert. Diese wird eingesetzt, um Objekte (Produkte, Behälter, Maschinen, Fahrzeuge und mehr) zu lokalisieren und identifizieren. Diese Objekte nehmen Informationen ihrer Umgebung auf und können über eindeutige Adressierungen angesprochen werden. Damit können Prozesse effektiver und effizienter gestaltet und gesteuert werden.

Das „Physical Internet" ist ein offenes, standardisiertes weltweites Güter-Transportsystem, basierend auf physischer, digitaler und operativer Interkonnektivität durch Protokolle, Schnittstellen und Modularisierung. Eine anbieter-, branchen- und grenzüberschreitende Standardisierung ist dafür Voraussetzung. Analog dem Konzept des digitalen Internets werden Materialflüsse vernetzt und virtualisiert. Durch standardisierte Transportbehälter wird eine maximale Auslastung von Transportfahrten erreicht, freie Kapazitäten können besser genutzt werden.

Das Prinzip kann sowohl in der Intralogistik als auch in übergreifenden Transportketten angewandt werden. Selbststeuernde, autonome Systeme in Transport und Lager sind Bausteinen eines Physical Internet. Durch gemeinsame Nutzung von Transportkapazitäten, Lagerstätten, Hubs und Übergabepunkten können ökonomische (kürzere Transportzeiten, geringere Personalkosten) wie auch ökologische (Reduktion von Verkehr und Emissionen) Nutzen erzielt werden.

Smart Logistics kann somit die Effizienz von Logistikketten verbessern und Wachstum durch neue Geschäftsmodelle erreichen. Die Potentiale liegen in der

- verbesserten Steuerung von Prozessen durch transparente Echtzeitdaten

- dynamischen, situationsbezogenen Gestaltung von Prozessen im Sinne einer adaptiven Selbststeuerung

- Nutzung von Synergien durch gemeinsame Nutzung von Kapazitäten, ermöglicht durch neutrale Plattformen

- verbesserten Entscheidungsfindung durch die Analyse umfangreicher Daten (Data Analytics), in Verbindung mit lernenden System

- Möglichkeit einer flexiblen, kundenspezifischen Anpassung von Produkten, Services und Abläufen

- Individualisierung von Designs, Konfiguration, Bestellung, Planung, Produktion und Betrieb unter wirtschaftlichen Bedingungen

- effektiven Zusammenarbeit von Mensch und Maschine und daraus resultierenden neue Möglichkeiten zur Arbeitsgestaltung und Kompetenzentwicklung
- Erschließung von Wertschöpfungspotentialen durch neue Dienstleistungen und Geschäftsmodelle der Logistik

\section{Voraussetzungen für den effektiven Einsatz von Smart Logistics}

Im Rahmen des Projektes SME4.0 - „Industry4.0 for SMEs Smart Manufacturing and Logistics for SMEs in an X-to-order and Mass Customization Environment" wurden explorativ Voraussetzungen für den effektiven Einsatz von Smart Logistics erhoben [7] und anschließend drei Clustern zugeordnet.

Als Erhebungsinstrument wurden hierbei strukturierte Expertenworkshops gewählt, welche basierend auf vordefinierten methodischen Richtlinien durchgeführt wurden [11]. Die Forschungspartner waren die Montanuniversität Leoben (Österreich), die Freie Universität Bozen (Italien), die Technische Universität Košice (Slowakei), das Worcester Polytechnic Institute (USA) und die Chiang Mai Universität (Thailand). Insgesamt wurden im Jahr 2017 von den Forschungsteams sechs Expertenworkshops mit 37 Unternehmen und 67 Experten durchgeführt und somit kumulativ 548 Expertenmeinungen für die nachfolgende qualitative Inhaltsanalyse gesammelt [12]

Nachfolgend werden die zentralen Ergebnisse der Studie kurz dargestellt [13].

Als wesentliche Voraussetzungen im Cluster "Intelligente und schlanke Lieferketten " wurden die Implementierung von Maßnahmen zur Erhöhung der Reaktionsfähigkeit bzw. Flexibilität der Produktion und Logistik (z.B. durch verbesserte Planungstools) und Strategien zur Reduktion von Kosten (z. B. Lean-Management-Initiativen, kollaborative Transportoptimierung und mehr) genannt. Wesentlich hierbei sind auch Methoden zur Steigerung der Robustheit (z.B. durch eine schnelle Identifikation und Reduktion von Störungen im Materialfluss) und der Transparenz (z. B. durch Echtzeit-Feedback des Auftragsfortschrittes) der gesamten Lieferkette. Weiters wurden die Ausbildung von qualifiziertem Fachpersonal sowie die Unterstützung des Top-Managements bei der Implementierung von neuen Technologien als wichtige Voraussetzungen genannt.

Aus dem Cluster "Intelligente Logistik mittels moderner Informations- und Kommunikationstechnologie" lassen sich im Wesentlichen Maßnahmen zur Herstellung einer verbesserten Konnektivität (z.B. durch eine gemeinsame Datenbasis, durch standardisierte Kommunikationsprotokolle und Schnittstellen und mehr) sowie Strategien zur Erhöhung der Datensicherheit ableiten. Zusätzlich spielt auch die Automatisierung von repetitiven Prozessen (z.B. automatisierte Bestellprozesse) sowie die Implementierung von benutzerfreundlicher Software (z. B. für laufende Bestandsanalysen) eine vorrangige Rolle.

Der Cluster "Intelligente Logistiksysteme und Transportmittel“ enthält schwergewichtige Maßnahmen zur Reduktion der manuellen Arbeitsleistung in Logistiksystemen (z.B. durch automatisierte Etikettierung, automatisierte Kommissionierung und Lieferung, automatisierte 
Lagerung) sowie den potentiellen Einsatz von fahrerlosen Transportsystemen (AGVs) und moderner Lagerverwaltungssoftware (z. B. mit fortgeschrittenen Optimierungsalgorithmen).

\section{Zusammenfassung und Ausblick}

Smart Logistics ist ein Kernelement von Digitalisierung in der industriellen Wertschöpfung, wie sie durch das Konzept Industrie 4.0 beschrieben wird. Sie basiert auf agilen Kooperationsnetzwerken sowie einer organisatorischen und informatorischen Vernetzung und ermöglicht intelligente und schlanke Lieferketten.

Cyber-physische Systeme (CPS), das Internet der Dinge (IoT) sowie das Physical Internet sind spezifische Konzepte von Smart Logistics, die die Bildung von (teil-)autonomen Systemen und Prozessen ermöglichen und damit die Effizienz von Logistikketten verbessern. Anbieterneutrale Plattformen für logistische Leistungen (Transporte, Lagerung, Verpackung, Materialfluss- und Informationsflusssteuerung und mehr) tragen zu einer effektiveren Ressourcennutzung bei und ermöglichen neue Geschäftsmodelle. Data Analytics bietet Werkzeuge für eine verbesserte Entscheidungsfindung.

Mit der weitgehend lückenlosen Verfolgung von Materialflüssen durch Verfahren der automatisierten Identifikation und Lokalisierung, der Entwicklung von selbststeuernden, autonome Systeme in Transport und Lager sowie der verstärkten Nutzung von Verfahren der Datenanalyse sind erste Schritte zu einer Smart Logistics getan worden.

Herausforderungen der Zukunft sind die anbieter-, branchen- und grenzüberschreitende Standardisierung von Systemen und Schnittstellen in Material- und Informationsflüssen, die Definition von Business Cases zum Nachweis der Sinnhaftigkeit von Technologieinvestitionen, die Entwicklung von Modellen der organisationischen Integration im Umfeld neuer Technologien, die Gewährleistung der Sicherheit für Menschen und Daten (Cyber Security) sowie die Entwicklung und Förderung der notwendigen Kompetenzen in der beruflichen Aus- und Weiterbildung.

Danksagung. Die empirische Erhebung wurde im Zuge des Projektes SME4.0 "Industry4.0 for SMEs - Smart Manufacturing and Logistics for SMEs in an X-toorder and Mass Customization Environment" durchgeführt. "This project has received funding from the European Union's Horizon 2020 research and innovation program under the Marie Skłodowska-Curie grant agreement No. 73471."

Funding. Open access funding provided by Montanuniversität Leoben.

Open Access Dieser Artikel wird unter der Creative Commons Namensnennung 4.0 International Lizenz (http://creativecommons.org/licenses/ by/4.0/deed.de) veröffentlicht, welche die Nutzung, Vervielfältigung, Bearbeitung, Verbreitung und Wiedergabe in jeglichem Medium und For- mat erlaubt, sofern Sie den/die ursprünglichen Autor(en) und die Quelle ordnungsgemäß nennen, einen Linkzur Creative Commons Lizenz beifügen und angeben, ob Änderungen vorgenommen wurden.

\section{Literatur}

1. Statista - Das Statistik-Portal: Welche Anwendungsbereiche von Industrie 4.0 sind heute bzw. werden zukünftig in Unternehmen stark ausgeprägt sein?, https://de.statista.com/statistik/daten/studie/ 605446/umfrage/anwedungsbereiche-von-indsutrie-40-heute-undzukuenfitg-in-der-dach-region/ (01.11.2018)

2. Bosch, G.; Bromberg, T.; Haipeter, T.; Schmitz, J.: Industrie und Arbeit 4.0. Befunde zu Digitalisierung und Mitbestimmung im Industriesektor auf Grundlage des Projekts „Arbeit 2020“, IAQ-Report: Aktuelle Forschungsergebnisse aus dem Institut Arbeit und Qualifikation, 2017, Nr. 04/2017, S. 1-24

3. ten Hompel, M.: Logistik 4.0, in Bauernhansl, T.; ten Hompel, M.; Vogel-Heuser, B. (Hrsg.): Industrie 4.0 in Produktion, Automatisierung und Logistik: Anwendung, Technologien, Migration, 1. Aufl., Wiesbaden (u.a.): Springer, 2014, S. 615-624

4. Kagermann, H.; Helbig, J.; Hellinger, A.; Wahlster, W.: Deutschlands Zukunft als Produktionsstandort sichern: Umsetzungsempfehlungen für das Zukunftsprojekt Industrie 4.0, Abschlussbericht des Arbeitskreises Industrie 4.0, 2013, Nr. 04, S. 1-116

5. Hermann, M.; Pentek, T.; Otto, B.: Design Principles for Industrie 4.0 Scenarios, in Bui, T. X.; Sprague R. H. (eds.): Proceedings of the 49th Annual Hawaii International Conference on System Sciences, Kauai, Hawaii, 2016, Piscataway: IEEE, 2016, S. 3928-3937

6. Bechtold, J.; Lauenstein, C.; Kern, A.; Bernhofer, L.: Industrie 4.0 Eine Einschätzung von Capgemini Consulting: Der Blick über den Hype hinaus, Capgemini Consulting, 2014, S. 1-116

7. Rauch, E.; Matt, D. T.; Brown, C. A.; Towner, W.; Vickery, A.; Santiteerakul, S.: Transfer of Industry 4.0 to Small and Medium Sized Enterprises, in Peruzzini, M.; Pellicciari, M., Bil, C.; Stjepandić, J., Wognum, N. (eds.): Advances in Transdisciplinary Engineering. Volume 7: Transdisciplinary Engineering Methods for Social Innovation of Industry 4.0, 2018, Nr. 07, S. 63-71

8. Bauernhansl, T.: Die Vierte Industrielle Revolution - Der Weg in ein wertschaffendes Produktionsparadigma, in Bauernhansl, T.; ten Hompel, M.; Vogel-Heuser, B. (Hrsg.): Industrie 4.0 in Produktion, Automatisierung und Logistik: Anwendung, Technologien, Migration, 1. Aufl., Wiesbaden (u. a.): Springer, 2014, S. 5-34

9. Broy, M.: Cyber-Physical Systems - Wissenschaftliche Herausforderungen Bei Der Entwicklung, in Broy,M. (Hrsg.): Cyber-Physical Systems: Innovation Durch Software-Intensive Eingebettete Systeme, 1. Aufl., Berlin (u. a.): Springer, 2010, S. 17-31

10. Lee, E. A.: CPS foundations, in Sapatnekar, S. (ed.): Proceedings of the 47th Design Automation Conference, Anaheim, California, 2010, New York: ACM Special Interest Group on Design Automation, 2010, S. 737-742

11. Krueger, R. A.; Casey, M. A.: Focus groups: A practical guide for applied research, 5. ed., Thousand Oaks: SAGE, 2015

12. Mayring, P.: Qualitative Inhaltsanalyse: Grundlagen und Techniken, 12. Aufl., Weinheim: Beltz, 2015

13. Die vollständigen Ergebnisse der Studie werden im nachfolgenden Artikel dargestellt: Dallasega, P.; Woschank, M.; Ramingwong, S.; Tippayawong, K. Y.; Chonsawat, N.: Development of Requirements for the Implementation of Smart Logistics in SMEs, Proceedings of the International Conference on Industrial Engineering and Operations Management, Bangkok, Thailand, 2019 